\title{
A SURVEY: PARTICLE SWARM OPTIMIZATION BASED ALGORITHMS TO SOLVE PREMATURE CONVERGENCE PROBLEM
}

\author{
Bahareh Nakisa, Mohd Zakree Ahmad Nazri, \\ Mohammad Naim Rastgoo and Salwani Abdullah
}

Faculty of Information Science and Technology, Universiti Kebangsaan Malaysia 43600 Bangi, Selangor, Malaysia

Received 2014-02-11; Revised 2014-04-18; Accepted 2014-04-24

\begin{abstract}
Particle Swarm Optimization (PSO) is a biologically inspired computational search and optimization method based on the social behaviors of birds flocking or fish schooling. Although PSO is represented in solving many well-known numerical test problems, but it suffers from the premature convergence. A number of basic variations have been developed due to solve the premature convergence problem and improve quality of solution founded by the PSO. This study presents a comprehensive survey of the various PSO-based algorithms. As part of this survey, we include a classification of the approaches and we identify the main features of each proposal. In the last part of the study, some of the topics within this field that are considered as promising areas of future research are listed.
\end{abstract}

Keywords: Particle Swarm Optimization (PSO), Premature Convergence, Diversity Guided Search

\section{INTRODUCTION}

PSO is one of the evolutional optimization methods and can solve many optimization problems that are encountered in various fields of technology such as switched reluctance motors (Balaji and Kamaraj, 2011), reduction of key search space of vigenere cipher (Sivagurunathan and Purusothaman, 2011), analog circuit (Kumar and Duraiswamy, 2012), controlling power systems (Mauryan et al., 2012) and etc. This method because of the simple concept and easy implementation has developed fast in recent years. A major problem with PSO is Premature Convergence (PC) especially in the case of multi-modal optimization problem, which results in great performance loss and sub-optimal solutions. The problem with premature convergence will always persist, since we obviously must check the whole search-space in order to ensure that a result is not sub-optimal. In spite of this fact and although the goals of maintaining high diversity and obtaining fast convergence is partially contradicting, it makes perfectly good sense to try to improve the optimization algorithm, in order to avoid sub-optimal solutions more frequently. Recently, there are several modifications from original PSO. This study provides the first survey of this study, attempting to classify these proposals that could be followed in the future by researchers in this area.

\section{PARTICLE SWARM OPTIMIZATION (PSO)}

Kennedy and Eberhart (1995) introduced particle swarm optimization that was based on social behavior of fish schooling or bird flocking. Particle swarm optimization by imitating from the social behavior of bird flocking, initializes a population of particles that simulates a flock of birds. The particles that each is represented as a solution are spread out in the search space randomly and search for finding the optimal or near optimal solution by generating new solutions. Each particle is represented with

Corresponding Author: Bahareh Nakisa, Faculty of Information Science and Technology,

Universiti Kebangsaan Malaysia 43600 Bangi, Selangor, Malaysia 
its position that is as a set of coordinates, which describes a point in a search space and its velocity and it's best past position achieved so far. At each iteration of the algorithm, particles in their current positions are evaluated through fitness function and if the value of fitness function is better than any that is found so far, it is stored as the best position called $\mathrm{P}_{\text {best }}$. The particle with the closest position to the goal gets the highest value in fitness function and is storedas. $\mathrm{P}_{\text {best }}$ After that, the next position that particle has to go and also its velocity is calculated by the following formula:

$$
\begin{aligned}
& \mathrm{v}_{\mathrm{i}+1}=\omega \mathrm{v}_{\mathrm{i}}+\mathrm{c}_{\mathrm{p}_{\mathrm{p}}}\left(\mathrm{p}_{\text {best }}-\mathrm{x}_{\mathrm{i}}\right)+\mathrm{C}_{\mathrm{g}} \mathrm{r}_{\mathrm{g}}\left(\mathrm{G}_{\text {best }}-\mathrm{x}_{\mathrm{i}}\right) \\
& \mathrm{x}_{\mathrm{i}+1}=\mathrm{v}_{\mathrm{i}+1}+\mathrm{x}_{\mathrm{i}}
\end{aligned}
$$

Where:

$$
\begin{aligned}
& \mathrm{X}_{\mathrm{i}} \in \mathrm{R}^{\mathrm{n}}=\text { The position of particle in the search space at } \\
& i^{\text {th }} \text { iteration } \\
& \mathrm{v}_{\mathrm{i}} \in \mathrm{R}^{\mathrm{n}}=\text { The velocity of particle at } i \text { th iteration } \\
& p^{\text {best }}=\text { The best position of particle achieved so far } \\
& g^{\text {best }}=\text { The best position of the particle in the swarm } \\
& \text { system that is achieved so far. } \\
& r_{p}, r_{g}=\text { Arethe random numbers between }(0,1) \\
& \text { generated at each iteration randomly for each } \\
& \text { particle } \\
& \mathrm{c}_{\mathrm{p}}, \mathrm{c}_{\mathrm{g}}=\text { Called acceleration coefficients } \\
& \omega=\text { Discussed as inertia weigh }
\end{aligned}
$$

\section{PSO-BASEDALGORITHMS FOR PREMATURECONVERGENCE PROBLEM}

Although PSO is one of the good techniques to find a good solution much faster than the other algorithms, it suffers from the premature convergence. It means that as the time in the PSO increases, the particles' momenta reduce and they tend to converge to a single point. Although convergence is an eligible property, PSO may fail to find the global optima when the problem is complicated and complex. Convergence property may cause the swarm to be trapped into one of them and not be able to explore other promising areas. In order to apply the PSO strategy for solving premature convergence problems, it is obvious that the original scheme has to be modified. In this study, we surveyed the most popular different techniques that solved this problem from 1989 till 2013. In the next subsections, the PSO-based algorithms are discussed.

\subsection{Modification of PSO}

Modifying a parameter may cause a large effect on the performance. There is a method that is proposed by Shi and Eberhart (1999; 2001) and increases the diversity in PSO by tuning inertia weight. Author said that by linearly decreasing the inertia weight value, the PSO tends to initially have more global search and then exploits more than the beginning of the search. The result shows that the PSO convergence is faster at the beginning and the convergence speed decreases when reaching the optima but the PSO suffers from the global search ability at the end and this is a drawback to escape from the local optima.

Another method that tunes the inertia weight is Fuzzy Logic Strategy that is proposed by Salehizadeh et al. (2009) named LOAPSO. They found that the tuning the PSO parameter has a great influence on the performance. This method adjusts dynamically with the acceleration coefficients during the optimization process.

Yadmellat et al. (2009) proposed a new Fuzzy tuned Inertia weight Particle Swarm Optimization (FIPSO) that remarkably outperforms the previous fuzzy. In this method the inertia weigh is dynamically adjust based on the average velocity and the current iteration.

One of the interesting approaches that have the ability to overcome the problem of premature convergence is ARPSO (Riget and Vesterstrom, 2002). ARPSO by utilizing the PSO as attraction phase and another formula as repulsion phase can maintain the diversity of PSO. This method initially measures the diversity and specifies the lower bound and upper bound, of diversity of population. In the attraction phases, the swarm moves toward each other based on the PSO formula. The movement of particles toward each other causes the population diversity decrease and when the diversity sees the $d$ !"\#, it will switch to the repulsion phase. Particles in the repulsion phase are sent away from each other based on a specific formula until the diversity reaches the upper bound. Then the algorithm switch back to the attraction phase again and the same process continues iteratively until particles reach the global optimum.

There is a new version of ARPSO called ATRE-PSO that is used in this study. This method is a simple extension of ARPSO that is proposed by Pant et al. $(2007 ; 2008)$ that has assumed a third phase called in between phase or the phase of positive conflict. As described before when the diversity falls below the lower bound. The algorithm switches to the repulsion phase and finally when the value of diversity reaches above of the upper bound we come back to the attraction phase 
but the diversity is placed between the two predefined thresholds many times. For this situation authors introduced a new phase, which is activated when the diversity is placed between lower bound and upper bound. In other words, when the population of diversity is lower than upper bound and greater than the lower bound the algorithm switches to the third phase. This phase is neither a complete attraction nor a complete repulsion. The particle that is attracted by its own best position is found so far and repelled by the best particle among all particles and by this method there is a balance between attraction and repulsion.

$\mathrm{Lu}$ and $\mathrm{Yu}$ (2012) proposed PSO-CAR which is based on an animal foraging strategy. This method creates a balance between the diversity and the convergence during the process. In the other words, firstly the particles should be grouped to ensure the convergence of the swarm and secondly they have to be self-centered that maintain a high diversity of the search.

Jie et al. (2012) proposed a new method that avoids the premature convergence. In this method the relationship between the particles and the optimal position is adjusted. In the other words, the distance between the particle and the optimal position is measured and if it is smaller than the certain radius the particle tend to move toward the opposite direction of optimal position, which is the repulsive force. The results show that the diversity among the particles is high enough to find the solution.

Krink et al. (2002) proposed a new approach that is called Spatial Extension PSO (SEPSO). In this method each particle has a volume, so each two particles with this volume check for collision. If they have collision, the action can be taken to make them bounce off from one another. The important issue in this method is to specify the direction of each particle for bouncing away from each other and with what speed. There are three strategies: The first one is random bouncing that particles in random direction sent away from the collision with the old velocity. The second one is Realistic physical bouncing and the third one is Simple velocity line bouncing that means particles move in the direction of the old velocity-vector, but with the scaled speed. However, studies in (Monson and Seppi, 2006) reported that by reducing the radius after every collision could maintain the diversity high as the time progress especially in multimodal functions.

Peram et al. (2003) introduced a new algorithm (FDR-PSO) that attracts the particles to move toward the nearby particles with higher fitness instead of global best particle achieved so far. This method adds a new term into the velocity component that also attracts the particles into the previous best experience of the "best nearest" neighbor. The new algorithm is shown to outperform PSO on many benchmark problems, being less susceptible to premature convergence and less likely to be stuck in local optima.

Ostadmohammadi et al. (2013) proposed a new method namely TPSO to increase the diversity. This method contains two approaches. In the first approach the diversity is controlled during the process of searching. The second approach introduce the new social interaction among the particles instead of previous selfcognitive ability of the swarm that help the particles to exploits the neighborhood in more effective way. In addition, this method applies the collision operator to enhance the diversity among the stronger and weaker particles. TPSO is compared with DMS-PSO- SHS, CLPSO, FDR-PSO and UPSO and he statistical results and convergence graphs demonstrate that TPSO reaches better quality of solution while it has relatively quicker convergence rate.

\subsection{PSO with Mutation}

Some researchers state that by adding a mutation operator to PSO the possibility is prepared to enhance the performance (Ratnaweera et al., 2004). There are two types of mutation operators: Particle position and Particle velocity. In the first mutation (TVAC), as the time progress if the global best solution does not improve then a random particle (mutated) and move to the random location in the search space. The second (SOH-PSO), the parameter varies during the optimization run. For the optimal solution the cognitive solution reduced while the social component increases as the time progress. This method by integrating TVAC and SOH-PSO can avoid the premature convergence in the early stages and promote convergence toward the global optimum solution.

Stacey et al. (2003) add a mutation operator. By applying this method tries to escape from the local optima and speed up the convergence. There is a global best particle among the particles that attracts all the particles toward itself. Thus mutate a single particle and if the new mutated particle becomes the new global best one all particles go away from the current location and they can escape from the local optima. This method is based on the particle position.

Another method that is based on the particle position mutation was proposed by Krohling (2005). There are two regimes in this method: For the first case, if there is 
progress in the fitness value from iteration to iteration, then particles move according to the Gaussian formula. Otherwise, it will switch to the second case. In the second phase, there is no improvement in the fitness value. Thus, their velocity speed is equal to zero and they stop in one area of the search space. In this case, they have to jump to a new position according to new formula, which may help them to escape from the local optima.

Li et al. (2007) proposed a method that prevents to fall into local optima by a Cauchy mutation operator called FPSO. FPSO introduces a Cauchy mutation for the position and velocity equation to jump out of the local optima. Cauchy mutation operator contains new formulas for position and velocity that every particle calculates its new position and velocity based on these formulas and also the original formulas. Each of these new positions and velocities has a smaller fitness value selected as a new one.

Ratanavilisagul and Kruatrachue (2011) proposed a method based on the FPSO that by using the crossover method could solve the premature convergence problem. In this method, the crossover updates position using position value in each dimension from the group best position or its previous best position. Both update can enhance the global search and retain local search ability. The result shows that the method without the crossover easily trop into the local optima in all functions. Therefore, the crossover operation selective version outperforms the nonselective version and increases the diversity.

Wang and Liu (2007a) introduced another version of PSO with Cauchy mutation. There is a specific formula for Cauchy mutation and it is used for global best particle for $\mathrm{N}$ times. After that, it compares the new global best particle with the last global best particle that is found by the PSO formula. If the fitness of the new global best particle, is smaller than the last global best particle then that replaces it. The result shows that this method has a good performance for the multi-modal problems.

There is a Gregarious Particle Swarm method (G-PSO) that was presented by Pasupuleti and Battiti (2006). In GPSO, The population moves toward the global best position and when they trap into the local optima, each particle is re-initialized with random velocity. This reinitialization occurs when the Euclidean distance between the current position and the global best position is less than the predefined threshold. This re-initialization is guided the swarm to escape from the local optima.

Thangaraj et al. (2009) proposed a new diversity guided Particle Swarm Optimization algorithm (PSO) named beta mutation PSO. This method contains two phases namely: Attraction phase and mutation phase. The attraction phase is like basic PSO and in the mutation phase the position vector is based on the Beta Distributed Mutation (BDM) operator. When the diversity among the particles reach below than the certain value then it switch to the mutation phase to increase the diversity.

\subsection{PSO with Opposite-Based Strategy}

Wang et al. (2007b) proposed the other method for solving the premature convergence and entrap into local optima. This method uses opposite-based PSO strategy to calculate the opposite position of each particle at each iteration and if the opposite has a better fitness value will be replaced by the original one. By applying the cauchy mutation on the best particle among all the particles in each generation, OPSO can find the better solution than the PSO. OPSO has a faster convergence on unimodal problems and has a better ability to search globally on multi-modal problems when it is compared with the original PSO.

Liang et al. (2004) Proposed PSOs with new learning strategies and CLPSO (Liang et al. 2006) where each dimension of a particle learned from just one particle's historical best information, while each particle learned from different particles' historical best information for different dimensions.

Wu et al. (2008) proposed a new method based on CLPSO namely OCLPSO. In this method not only learn from other exemplar for different dimension but also learn from their opposite particles. In the other words, firstly, two particles are selected from the population and their fitness and their opposite are compared then the fitter particles are used as exemplar for that dimension. The results represent that OCLPSO has a better performance than CLPSO in 10 benchmark function.

Chi and Cai (2010) proposed a new method that called the Particle Swarm Optimization with Oppositionbased Disturbance (PSOOD) to adopt the OppositionBased Disturbance (OBD) procedure. This method based on the OBD, disturb the position of particles when the personal best position is updated that increase the diversity of population to find the better solution.

\subsection{PSO Hybrids}

One of the approaches that result in an escape from local optima is hybridizing the PSO with breeding and subpopulations that are based on the standard Genetic algorithm (Lovbjerg et al., 2001). In this method each 
particle has a breeding probability and this probability specify which particle should be marked for breeding. There is a group of marked particle that we select two particles randomly for breeding and replace the parents with their offspring's. There are specific formulas for calculating the offspring position and velocity. Another thing is about the subpopulation that the population of the swarm is divided into sub-groups that there is a separate global best particle in each sub-group thus the parents can select from the same subpopulation or from the different subpopulations.

Yang et al. (2007) proposed a method that hybridizes the Genetic algorithm and PSO algorithm to enhance the diversity. This method manipulates the crossover, reproduction and mutation of GA with predetermined probability. In this method there are two stages: In the first stage the particles based on its best experience and its neighbor fly in the search space. In the second stage based on the GA, the selection, reproduction, crossover and mutation operation are applied on particles with predetermined probability. The result shows that the mutation plays a key role on the diversity of particles. The mutation operation is applied on particle based on its fitness.

Wang et al. (2008) combined the Novel MultiParent Crossover Operator (NMPCO) and a selfadaptive Cauchy mutation operator that called MPSO to avoid the premature convergence. NMSPO enhance the local search anility by using the full interaction between itself and other particles in leaner space. The Self-adaptive Cauchy mutation helps the particles to escape from the local optima and enhance the global search ability. The result shows that the MPSO in some cases entrap into the local optima due to the number of parents in the NMPSO may contain too much noise for the problem.

Tang and Zhao (2009) proposed a method by hybridizing the PSO with a novel Adaptive Mutation operator that called AMPSO. When the global best particle entrap into the local optima has no changes during the iteration, it means that it is entrapped into the local optima. Therefore by applying mutation into the global best particle in each generation it would be helpful to jump out from the local optima. AMPSO generate an adaptive mutation that dynamically adjusts the mutation size in terms of the size of current search space. Experimental studies on 8 well-known benchmark functions show that AMPSO performs better than PSO, PSO with Gaussian mutation and PSO (GPSO) with Cauchy mutation (CPSO) on most test functions.

Tang and Zhao (2010) proposed a hybrid PSO, namely LSPSO, to solve this problem by employing an adaptive local search operator. The main idea of LSPSO is to search the neighborhood of the global best particle with adaptive step size. The result shows that the performance of LSPSO is better than PSO, PSO with Gaussian mutation and PSO (GPSO) with Cauchy mutation (CPSO) on majority of test problems but still have premature convergence in one function and this is due to the applying pure mutation techniques.

Masrom et al. (2004) proposed a method that hybridizes the PSO with GA operators. In this study, a number of PSO hybrids combined with adaptive parameterization. The first algorithm combines both adaptive parameterizations to the crossover and mutation. The second and third algorithms use only one of the operations. The result among these PSO hybrids shows that the result with the adaptive mutation has the best. A combination of crossover and mutation in PSO has the better result than the inclusion of crossover on its own and worse than the mutation on its own.

\subsection{Multi-Swarm PSO}

Zhao et al. (2008) proposed DMS-PSO based on the new neighborhood topology. In this method the whole of the population are divided into small sized swarm. Each sub-swarm uses its own members to search for better regions in the search space. In order to increase the diversity these sub-swarms are regrouped frequently to exchange the information among all particles. A local search is combined with the algorithm to improve the overall algorithm's local searching ability. The DMS-LPSO is tested on a set of benchmark functions and the results show that the proposed algorithm can find reasonable solutions for all of the problems.

Li and Xiao (2008) proposed a new method named Multi-Best PSO (MBPSO). This method instead of using single global best position ( $\mathrm{g}_{\text {best }}$ ) and personal best position $\left(\mathrm{P}_{\text {best }}\right)$, it uses the multi $\mathrm{g}_{\text {best }}$ and multi $\mathrm{P}_{\text {best }}$. So in the course of searching, other best values can help the best value trapped by local optimum fly out of local position. MBPSO divided the whole population into the sub-swarms and then calculates the several $g_{\text {best }}$ and then combines all particles together and then calculates again taking the result as a new initial value.

DMS-PSO-SHS (Zhao et al., 2010) is an extension of DMS-PSO that divided the whole of the population into the small sub-swarm with dynamic size to adopt each one the population of the harmony search algorithm. This method based on the DMS-PSO, generate new harmonies according to the current personal best solution and the nearer personal best solution is replaced with a new harmony with better fitness. The DMS-PSO-SHS 
enables the particles to have more diverse exemplars to learn from after we frequently regroup the swarms and allow the harmonies to search in a larger potential space among different sub-populations.

Jie et al. (2010) proposed a Multi-swarm Particle Swarm Optimization (MPSO) to maintain the swarm diversity. This method applied a mixed local search behavior modes and information exchange among subswarms. When the premature convergence occurs in one sub-swarm then that particles should escape from the local area through the initialization their position in the search space.

\section{CONCLUSION}

Although PSO is one of the good techniques to find a good solution much faster than the other algorithms, it suffers from the premature convergence. In other words, when the number of iteration increases the quality of the solution by the PSO cannot improve and it converges to the local optima which is may not be the global optima. This study discussed the various PSO based algorithms and techniques employed to solve the premature convergence problem for achieving optimal performance. Then we have analyzed each of them in more detail. We have also proposed taxonomy to classify the current techniques reported in the specialized literature and we have provided a survey of approaches based on such taxonomy. Considering the current rate of growth of this area, we expect a lot of more activity within the next few years.

\section{REFERENCES}

Balaji, M. and V. Kamaraj, 2011. Particle swarm optimization approach for optimal design of switched reluctance machine. Am. J. Applied Sci., 8: 374-381. DOI: 10.3844/ajassp.2011.374.381

Mauryan, K.S.C., K. Thanushkodi and A. Sakthisuganya, 2012. Reactive power optimization using quantum particle swarm optimization. J. Comput. Sci., 8: 1644-1648. DOI: 10.3844/jcssp.2012.1644.1648

Chi, Y. and G. Cai, 2010. Particle swarm optimization with opposition-based disturbance. Proceedings of the 2nd International Asia Conference on Informatics in Control Automation and Robotics, March 6-7, IEEE Xplore Press, Wuhan, pp: 223226. DOI: 10.1109/CAR.2010.5456563.
Kumar, P.P. and K. Duraiswamy, 2012. An optimized device sizing of analog circuits using particle swarm optimization. J. Comput. Sci., 8: 930-935. DOI: $10.3844 /$ jcssp.2012.930.935

Kennedy, J. and R. Eberhart, 1995. Particle swarm optimization. Proceedings of IEEE International Conference on Neural Networks, Nov. 27-Dec. 1, IEEE Xplore Press, Perth, WA., pp: 1942-1948. DOI: $10.1109 /$ ICNN.1995.488968

Krink, T., J.S. VesterstrOm and J. Riget, 2002. Particle swarm optimisation with spatial particle extension. Proceedings of the Congress on Evolutionary Computation, May 12-17, IEEE Xplore Press, Honolulu, HI., pp: 1474-1479. DOI: 10.1109/CEC.2002.1004460

Jie, J., W. Wang, C. Liu and B. Hou, 2010. Multiswarm particle swarm optimization based on mixed search behavior. Proceedings of the 5th IEEE Conference on Industrial Electronics and Applications, Jun. 15-17, IEEE Xplore Press, Taichung, $\quad$ pp: 605-610. DOI: 10.1109/ICIEA.2010.5517044

Jie, Z., F. Chaozan, L. Bo and S. Fugui, 2012. An improved particle swarm optimization based on repulsion factor. Open J. Applied Sci., 2: 112115. DOI: 10.4236/ojapps.2012.24B027

Krohling, R.A., 2005. Gaussian particle swarm with jumps. Proceedings of IEEE Congress on Evolutionary Computation, Sept. 2-5, IEEE Xplore Press, pp: 1226-1231. DOI: 10.1109/CEC.2005.1554830

Li, C., Y. Liu, A. Zhou, L. Kang and H. Wang, 2007. A Fast Particle Swarm Optimization Algorithm with Cauchy Mutation and Natural Selection Strategy. Advances in Computation and Intelligence, Springer, ISBN: 978-3-540-74580-8, pp: 334-343.

Li, J. and X. Xiao, 2008. Multi-swarm and multi-best particle swarm optimization algorithm. Proceeding of the 7th World Congress on Intelligent Control and Automation, June 25-27, IEEE Xplore Press, Chongqing, pp: 6281-6286. DOI: 10.1109/WCICA.2008.4593876

Liang, J.J., A.K. Qin, P. Suganthan and S. Baskar, 2004. Particle swarm optimization algorithms with novel learning strategies. Proceedings of the IEEE International Conference on Systems Man and Cybernetics, Oct. 10-13, IEEE Xplore Press. DOI: 10.1109/ICSMC.2004.1400911 
Liang, J.J., A.K. Qin, P.N. Suganthan and S. Baskar, 2006. Comprehensive learning particle swarm optimizer for global optimization of multimodal functions. IEEE Trans. Evolut. Comput., 10: 281295. DOI: $10.1109 /$ TEVC.2005.857610

Lovbjerg, M., T.K Rasmussen and T. Krink, 2001. Hybrid particle swarm optimiser with breeding and subpopulations. Proceedings of the Computation Conference on, Genetic and Evolutionary Morgan Kaufmann, (EMM' 01), pp: 469-476.

Lu, S. and S. Yu, 2012. An improved particle swarm optimizer with attraction and repulsion. Proceedings of the 7th International Computing and Convergence Conference on Technology, Dec. 3-5, IEEE Xplore Press, Dalian, pp: 3193-3197. DOI: 10.1109/WCICA.2006.1712956

Masrom, S., M. Perak, I. Moser, J. Montgomery and S.Z.Z. Abidin et al., 2004. Hybridization of particle swarm optimization with adaptive genetic algorithm operators. Syst. Man Cyber., 34: 997-1006. DOI: 10.1109/TSMCB.2003.818557

Monson, C.K. and K.D. Seppi, 2006. Adaptive diversity in PSO. Proceedings of the 8th Annual Conference on Genetic and Evolutionary Computation, Jul. 08-12, ACM., New York, pp: 59-66 DOI: 10.1145/1143997.1144006

Ostadmohammadi, A.B., P. Mirzabeygi and M.S. Panahi, 2013. An improved pso algorithm with a territorial diversity-preserving scheme and enhanced exploration-exploitation balance. Swarm Evolut. Comput., 11: 1-15. DOI: 10.1016/j.swevo.2012.12.004

Pant, M., T. Radha and V.P. Singh, 2007. A simple diversity guided particle swarm optimization. Proceedings of the IEEE Congress on Evolutionary Computation, Sept. 25-28, IEEE Xplore press, Singapore. DOI: 10.1109/CEC.2007.4424896

Pant, M., R. Thangaraj and A. Abraham, 2008. Particle swarm optimization using adaptive mutation. Proceedings of the 19th International Workshop on Database and Expert Systems Application, Sept. 1-5, IEEE Xplore Press, Turin. DOI: 10.1109/DEXA.2008.70.

Pasupuleti, S. and R. Battiti, 2006. The Gregarious Particle Swarm Optimizer (G-PSO). Proceedings of the 8th Annual Conference on Genetic and Evolutionary Computation, Jul. 08-12, ACM., New York, pp: 67-74. DOI: 10.1145/1143997.1144007
Peram, T., K. Veeramachaneni and C.K. Mohan, 2003. Fitness-distance-ratio based particle swarm optimization. Proceedings of the IEEE Swarm Intelligence Symposium, Apr. 24-26, IEEE Xplore Press, pp: 174-181. DOI: 10.1109/SIS.2003.1202264

Ratanavilisagul, C. and B. Kruatrachue, 2011. Selective crossover base on fitness in multiswarm optimization. Proceedings of the International Conference on Emerging Trends in Computer and Image Processing, (CIP' 11), Bangkok, pp: 12-15.

Ratnaweera, A., S.K. Halgamuge and H.C. Watson, 2004. Self-organizing hierarchical particle swarm optimizer with time-varying acceleration coefficients. IEEE Trans. Evolut. Comput., 8: 240-255. DOI: 10.1109/TEVC.2004.826071

Riget, J. and J.S. Vesterstrom, 2002. A diversity-guided particle swarm optimizer-the arpso. Dept. Comput. Tech. Rep.

Salehizadeh, S., P. Yadmellat and M. Menhaj, 2009. Local optima avoidable particle swarm optimization. Preceedings of the IEEE Swarm Intelligence Symposium, Mar. 30-Apr. 2, IEEE Xplore Press, Nashville, TN., pp: 16-21. DOI: 10.1109/SIS.2009.4937839.

Sivagurunathan, G. and T. Purusothaman, 2011. Reduction of key search space of vigenere cipher using particle swarm optimization. J. Comput. Sci., 7: 1633-1638. DOI: 10.3844/jcssp.2011.1633.1638

Shi, Y. and R.C Eberhart, 1999. Empirical study of particle swarm optimization. Proceedings of the Congress on Evolutionary Computation, Jun. 6-9, IEEE Xplore Press, Washington, DC., DOI: 10.1109/CEC.1999.785511

Shi, Y. and R.C. Eberhart, 2001. Fuzzy adaptive particle swarm optimization. Proceedings of the Congress on Evolutionary Computation, IEEE Press Xplore, Seoul, pp: 101-106. DOI: 10.1109/CEC.2001.934377

Stacey, A., M. Jancic and I. Grundy, 2003. Particle swarm optimization with mutation. Proceedings of the Congress on, Evolutionary Computation CEC'03. Dec. 8-12, IEEE Xplore Press, DOI: 10.1109/CEC.2003.1299838.

Tang, J. and X. Zhao, 2009. Particle swarm optimization with adaptive mutation. Proceedings of the International Conference on Information Engineering, Jul. 10-11, IEEE Xplore Press, Taiyuan, Shanxi, pp: 234-237. DOI: 10.1109/ICIE.2009.59 
Tang J. and Zhao X., 2010. A hybrid particle swarm optimization with adaptive local search. J. Net., 5: 411-418.

Thangaraj, R., M. Pant and A. Abraham, 2009. A new diversity guided particle swarm optimization with mutation. Proceedings of the Nature and Biologically Inspired Computing, Dec. 9-11, IEEE Xplore Press, Coimbatore, pp: 294-299. DOI: 10.1109/NABIC.2009.5393723

Wang, H., H. Li, Y. Liu, C. Li and S. Zeng, 2007a. Opposition-based particle swarm algorithm with cauchy mutation. Proceedings of the IEEE Congress on Evolutionary Computation, Sept. 2528, IEEE Xplore Press, Singapore, pp: 4750-4756. DOI: 10.1109/CEC.2007.4425095

Wang, H., Y. Liu, C. Li and S. Zeng, 2007b. A hybrid particle swarm algorithm with cauchy mutation. Proceedings of the IEEE Swarm Intelligence Symposium, Apr. 1-5, IEEE Xplore Press, Honolulu, HI, pp: 356-360. DOI: 10.1109/SIS.2007.367959

Wang, H., Z. Wu, Y. Liu and S. Zeng, 2008. Particle swarm optimization with a novel multi-parent crossover operator. Proceedings of the 4th International Conference on Natural Computation, Oct. 18-20, IEEE Xplore Press, Jinan, pp: 664668. DOI: $10.1109 /$ ICNC.2008.643

Wu, Z., Z. Ni, C. Zhang and L. Gu, 2008. Opposition based comprehensive learning particle swarm optimization. Proccedings of the 3rd International Conference on Intelligent System and Knowledge Engineering, Nov. 17-19, IEEE Xplore Press, Xiamen, pp: 1013-1019. DOI: 10.1109/ISKE.2008.4731078
Yadmellat, P., S.M.A. Salehizadeh and M.B. Menhaj, 2009. A new fuzzy inertia weight particle swarm optimization. Proceedings of the Conference on Computational Intelligence and Natural Computing, Jun. 6-7, IEEE Xplore Press, Wuhan, pp: 507-510. DOI: 10.1109/CINC.2009.180

Yang, B., Y. Chen and Z. Zhao, 2007. A hybrid evolutionary algorithm by combination of pso and ga for unconstrained and constrained optimization problems. Proceedings of the International Conference on Control and Automation, May 30Jun. 1, IEEE Xplore Press, Guangzhou, pp: 166170. DOI: 10.1109/ICCA.2007.4376340.

Zhao, S.Z., J.J. Liang, P.N. Suganthan and M.F. Tasgetiren, 2008. Dynamic multi-swarm particle swarm optimizer with local search for large scale global optimization. Proceedings of the IEEE Congress on Evolutionary Computation, Jun. 1-6, IEEE Xplore Press, Hong Kong, pp: 3845-3852. DOI: $10.1109 / C E C .2008 .4631320$

Zhao, S.Z., P.N. Suganthan and S. Das, 2010. Dynamic multi-swarm particle swarm optimizer with sub-regional harmony search. Proceeding of the IEEE Congress on the Evolutionary Computation, July 18-23, IEEE Xplore Press, Barcelona, $\quad$ pp: $1-8$. DOI: 10.1109/CEC.2010.5586323 